\title{
Model Reduction Methods for Rotor Dynamic Analysis: A Survey and Review
}

\author{
Matthew B. Wagner, ${ }^{1}$ Amir Younan, ${ }^{1}$ Paul Allaire, ${ }^{1}$ and Randy Cogill² \\ ${ }^{1}$ Department of Mechanical and Aerospace Engineering at University of Virginia, 122 Engineer's Way, P.O. Box 400746, Charlottesville, \\ VA 22904-4746, USA \\ ${ }^{2}$ Department of Systems and Information, University of Virginia, 151 Engineer's Way, P.O. Box 400747, Charlottesville, \\ VA 22904-4747, USA
}

Correspondence should be addressed to Matthew B. Wagner, mbw7p@virginia.edu

Received 30 September 2010; Accepted 21 December 2010

Academic Editor: Jerzy Sawicki

Copyright (๑) 2010 Matthew B. Wagner et al. This is an open access article distributed under the Creative Commons Attribution License, which permits unrestricted use, distribution, and reproduction in any medium, provided the original work is properly cited.

The focus of this literature survey and review is model reduction methods and their application to rotor dynamic systems. Rotor dynamic systems require careful consideration in their dynamic models as they include unsymmetric stiffness, localized nonproportional damping, and frequency-dependent gyroscopic effects. The literature reviewed originates from both controls and mechanical systems analysis and has been previously applied to rotor systems. This survey discusses the previous literature reviews on model reduction, reduction methods applied to rotor systems, the current state of these reduction methods in rotor dynamics, and the ability of the literature to reduce the complexities of large order rotor dynamic systems but allow accurate solutions.

\section{Introduction}

There has been a long-standing interest in rotor dynamic modeling that has evolved since the first and incorrect rotor dynamic analysis by Rankine in 1869 . The need to understand the system response to design predictable, low maintenance, cost-effective, and optimal systems has driven rotor dynamacs to often model large-order complex rotor system models. The models contain more intricate geometric complexities, bearings, seals, and attached components such as disks, blades, fans, and couplings. Some models involve relatively simple beam representations of the rotor on bearings that are represented as stiffness and damping. These models do not usually need reduction so they are not the topic covered here. The standard analysis of rotor systems includes critical speeds, stability, and unbalanced response $[1,2]$.

For many analyses it is also of interest to look at rotor systems with foundation coupling for response analysis, integration of the rotor with its full system, transient behavior, failure, and even fluid-structural effects. However, the varied nature of these models makes it computationally inefficient or near impossible to perform a rotor dynamic analysis on the system. Rotor dynamic models require careful consideration in their dynamic models as they include unsymmetric stiffness, localized nonproportional damping and frequency dependent gyroscopic effects.

Some examples of such complex rotor models can be seen in Figures 1 and 2. Figure 1 shows a complex rotor dynamic system of the low and high pressure shaft assemblies of a jet engine. In this figure it is apparent that the system is complex. The shafts are hollow and flexible; the shaft has complex geometry; there are multiple attached bladed disk assemblies and the bearings connect the two shafts together and to the engine casing and aircraft structure. Figure 2 is a model of a large alternator/flywheel train that is employed as part of the power system for the ALCATOR CMOD experiment at the MIT Plasma Fusion Center [3]. The alternator is used to provide peak pulse power of $100 \mathrm{MW}$ to the magnets employed in the fusion experiment. After being driven up to full speed over a long period of time by a $1491 \mathrm{~kW}$ motor, the alternator is rapidly decelerated from 


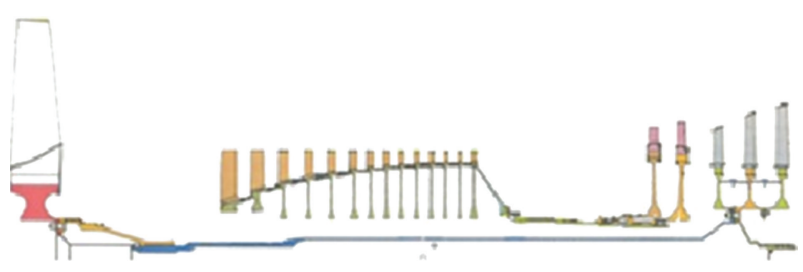

FIGURE 1: The low and high pressure shaft assemblies of a jet engine.

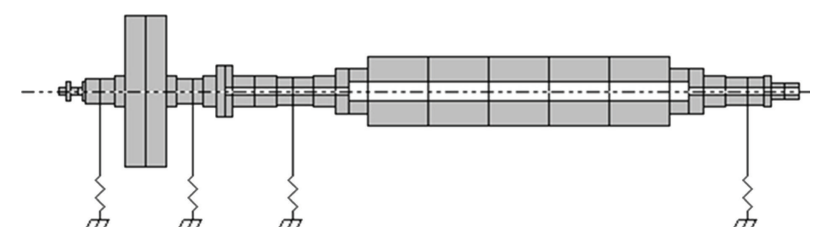

FIGURE 2: The model of a large alternator/flywheel train at the MIT Plasma Fusion Center.

static and dynamic and used for the system static and dynamic analyses. The static methods are typically based upon Guyan reduction and variations thereof. The dynamic methods commonly used are real and complex modal analysis, component mode synthesis, and other formulations of modal methods.

The literature on rotor dynamics supports the use of reduction methods from both controls and structures. Both attempt to handle unsymmetric stiffness, system damping, and gyroscopics through derivations for nonrotating selfadjoint problems. The literature consistently

(i) presents new variations of methods many of which are for very specific systems,

(ii) applies the reduction methods to small finite element models that do not actually need reduction,

(iii) often makes several assumptions to reduce the rotor beam dimensionality of the system and mitigate or eliminate the rotor dynamic effects before the presented reduction method is used,

(iv) lacks a full rotor dynamic analysis and the effects of various model reduction methods on the analysis,

(v) uses full order model results, which are small enough to analyze, to compare with reduced order model results.

The current literature does not provide a review on model reduction methods of rotor dynamic systems, nor clear comparisons between reduction method results. The literature needs to address the reduction of complex large order rotor dynamic models. When reducing large order models the analysis of the full order is not necessarily possible and the direct comparison with the reduced order models is not likely feasible. New reduction metrics, which are seldom discussed in the literature, need to be used to evaluate the ability of the reduction methods to meet criteria for reduced order models. These metrics provide insight into the ability of a reduction method, the chosen basis for reduction, and the order of the basis for reduction to accurately describe the dynamic system. Finally, there is the need for a comparison of the basis for which reduction should be performed to provide an accurate and computationally efficient model for a rotor dynamic system. 


\section{Previous Literature Reviews on Model Reduction}

This section of the paper gives an overview of previously published literature surveys on model reduction. These reviews are limited in scope, focusing on specific model reduction methods, and do not directly discuss application to rotor dynamic systems. The reviews are presented in chronological order by their publication date.

Noor [7] compiled an extensive survey of the mathematical aspects of model reduction methods applied to linear and nonlinear systems. Noor notes that prior to this review in 1994 the only survey papers on the subject of model reduction date back to the early 1980's and are limited in their scope, primarily focused on nonlinear problems. Noor provides good insights into model reduction, selection of global approximation vectors, guidelines for computational procedures, and an extensive review of applications in the literature. Noor [7] gives detailed discussions on the eigenvalues problem, nonlinear vibrations, initial/boundary value problems, sensitivity analysis, optimization and reanalysis of systems, and error estimation.

Noor's review [7] also gives some insights into expected future directions including efficient and automatic generation of global approximation vectors, reduction with system partitioning, and contact problems. However, the paper focuses on nonlinear problems and the selection of basis (mathematical depiction) for multiple parameter, nonconservative, operator splitting. techniques for application to various mathematical descriptions for problems. This review does not discuss applications to rotating machinery or the complications thereof.

Antoulas et al. [4] reviewed model reduction methods for large-scale systems that span a wide application from structural, dynamic, and controls problems not including rotor dynamic systems. The review is presented as a discussion of reduction as two categories: singular value decomposition and moment matching methods. This paper discusses and provides numerical comparisons of common methods of Singular Value Decomposition (SVD): balanced model reduction, approximate balanced reduction, singular perturbation, and Hankel norm approximation and Moment Matching methods: Lanczos procedure, Arnoldi procedure, Rational Krylov method. The conclusions of this paper are that the SVD-based methods provide the best results when considering the whole frequency range of a system. Moment matching methods have higher error norms but greatly reduce the computational costs and requirements.

The examples in [4] are small in order $(<400 \mathrm{DOF})$. The system characteristics and structure are briefly described and the way in which the structure of the system is handled by the reduction is not discussed: an important consideration when analyzing rotor dynamic systems. The paper does not discuss the application of these methods in previous literature nor rotor dynamic models. This paper also does not fully explain the reduction order, or basis size, in the comparisons between the reduction methods.

Gugercin and Antoulas [8] present a survey of model reduction methods via balanced truncation formulations.
This survey presents detailed mathematical discussions on 5 balancing methods and their corresponding error norms: Lyapunov, stochastic, bounded real, positive real, and frequency weighted balancing. This survey introduces a multiplicative-type error for positive real balancing and introduce a new algorithm for weighted balanced truncation that guarantees stability and $H_{\infty}$ bound on the error. This survey is limited in that it strictly defines the mathematical formulations of the balancing methods and has a limited discussion on the comparison of these methods and their respective norms.

$\mathrm{Qu}$ [6] discusses model order reduction techniques with applications in finite element analysis. Through this book $\mathrm{Qu}$ discusses static and dynamic condensation methods, modal analysis, iterative dynamic condensation methods, and substructuring with model reduction for both classically and nonclassically damped systems. Applications are used for both vibrational control, structural/dynamic analysis of a system, and modal testing for test analysis correlation between models and physical systems. This book provides an overview of model reduction via finite elements but does not discuss rotating systems and the complexities of reduction for these systems.

Antoulas [9] comprehensively discusses model order reduction techniques of Large-Scale Dynamical Systems. Through this book Antoulas discusses reduction methods for linear dynamical systems in both the time and frequency domains with methods of realization, rational interpolation, singular value decomposition, Krylov, and norm-based reduction methods. Case studies are presented for a wide range of structural, heat transfer, electronics, and other practical problems. This book provides a detailed mathematical framework and toolbox of model reduction methods applied to a wide array of mathematical models.

Ghafoor and Sreeram [10] review frequency weighted balanced model reduction methods. In this survey they discuss 5 methods that have been developed and provide a comparison between these methods. The methods include an extension of balanced truncation by Enns [11] with input, output or weightings on both input and output. Lin and Chiu's [12] method overcomes problems with system instability in double-sided weighting. Varga and Anderson [13] modified Lin and Chiu's method to account for no polezero cancellation in Lin and Chiu's method for controller design. They also review Wang's method [14] and a partial fraction expansion-based method developed by the authors.

The final conclusion of Ghafoor and Sreeram's review [10] is that Enns' method provides the best approximation compared to other techniques, but can yield unstable results. The other methods guarantee stability for the case of doublesided weighting but do not more accurately approximate the systems than Enns' method. Thus, a method that can guarantee stability and produce low approximation errors needs to be developed. A more detailed survey of the mathematical formulations of the methods discussed in [10] of frequency weighted methods was written [15].

The most recent overview of model reduction methods is discussed by Antoulas [16]. This paper gives a brief overview of model reduction as SVD- and Krylov-based 
methods as previously discussed in Antoulas's review [4]. Here they present a method that discusses the connection between these two methods-computing reduced systems obtained by balanced truncation using Krylov methods. They found that it was possible to reduce a system by balanced truncation through Krylov's method but found this to be computationally inefficient.

This current literature review is more limited in scope than some previous literature reviews in that it concentrates on linear time-dependent dynamical systems, specifically rotor dynamic systems. The emphasis of this review is on model reduction methods applied to rotor dynamic modeling, analysis, and control. This review gives an overview of rotor dynamic systems, modeling methods of these systems, reduction methods that have been previously developed and used in both the fields of structures and controls and how they apply to rotor dynamic systems, and a discussions of model reduction metrics. Discussions on the ability of these applied methods to rotor dynamic systems are discussed in relation to the typical unsymmetric bearing stiffness, gyroscopic, nonproportional bearing damping, and other nonsymmetric rotor or fluid stiffness, damping and mass matrices found in rotor systems. This review considers model reduction methods applied to both full rotor systems and the components within.

\section{Rotor Dynamic Systems}

A typical rotor system is comprised of a rotor, built-on parts, and a support system. The shaft or rotor is the main structure in a rotor dynamic system and can have many configurations in a system: single, multiple, concentric, and coupled and may be modeled as 1D beams or 3D solid elements. The built-on parts are attached to the rotor and include impellers, gears, fans, disks, and couplings. The support system connects the rotor to an external structure and includes bearings, seals, and squeeze-film dampers. Figures 1 and 2 show examples of a rotor dynamic system with these components.

Rotor systems are similar to other areas of vibrations but include some key differences that differentiate the analyses and methods for analyzing these systems. In the stationary reference frame these key differences include the frequencydependent gyroscopic effects and localized nonproportional damping which can also be frequency dependent to the running speed $\Omega$ that make the system non-selfadjoint and unsymmetric. In a dynamic interpretation this means that instead of having planar modes, as in most structural systems, rotor dynamic systems have complex modes that result from the inclusion of unsymmetric stiffness, nonproportional damping, gyroscopics, and frequency-dependent effects.

A rotor model represented in the rotating reference frame, can support unsymmetric geometry and the resulting gyroscopics, but proves to be computationally difficult in adding anisotropic bearings. In the rotating reference frame terms for rotating damping and centrifugal stiffening are included. However, as stated by Genta and Gugliotta [17],
TABle 1: Component contributions to rotor dynamic equation of motion; $\mathrm{S}=$ symmetric, $\mathrm{SS}=$ skew-symmetric, and US = unsymmetric.

\begin{tabular}{lcccc}
\hline & $\begin{array}{c}\text { Mass } \\
(\mathrm{M})\end{array}$ & $\begin{array}{c}\text { Damping (C) } \\
(\mathrm{S})\end{array}$ & $\begin{array}{c}\text { Gyroscopic } \\
(\mathrm{G})\end{array}$ & $\begin{array}{c}\text { Stiffness } \\
(\mathrm{K})\end{array}$ \\
\hline Shaft & $\otimes$ & $\otimes$ & $(\mathrm{SS})$ & $(\mathrm{S} / \mathrm{US})$ \\
\hline Impeller & $\otimes$ & & $\otimes$ & $\otimes$ \\
Gear & $\otimes$ & & $\otimes$ & $\odot(\mathrm{US})$ \\
Fan & $\otimes$ & & $\otimes$ & \\
Disk & $\otimes$ & & $\otimes$ & \\
Coupling & $\otimes$ & & $\otimes$ & $\odot(\mathrm{S})$ \\
\hline Rolling Bearing & & & & $\otimes(\mathrm{US})$ \\
Journal Bearing & & $\otimes(\mathrm{US})$ & & $\otimes(\mathrm{US})$ \\
Squeeze Film & $\odot$ & $\otimes(\mathrm{US})$ & & $\otimes(\mathrm{US})$ \\
Damper & & & & $\otimes(\mathrm{US})$ \\
Seals & $\bigcirc$ & $\otimes(\mathrm{US})$ &
\end{tabular}

the addition of centrifugal stiffening is only for the rotating reference frame and is not worth the computational costs when the model can be further discretized to yield accurate results as discussed for beam models. Rotating damping, damping associated with the rotor itself such as material damping, will typically reduce amplitude vibration in subcritical operation and can be destabilizing in supercritical situations. This damping is important to include as material or internal damping, friction in threaded shafts, intershaft dampers in multirotor machines. If there are external forces applied to the system these can be a function of the running speed, synchronous such as in the case of unbalance.

The equation of motion that describes a rotor system in stationary reference frame is [3]

$$
M \ddot{X}+\left(C+\left(C_{b}+G\right) \Omega\right) \dot{X}+\left(K+K_{b} \Omega\right) X=F+F_{\omega} .
$$

For the rotating reference frame is [18]

$$
M \ddot{X}+((C+G) \Omega) \dot{X}+\left(K+K_{b} \Omega-K_{c} \Omega^{2}\right) X=F+F_{\omega},
$$

where the mass $(M)$, damping $(C)$, bearing and/or rotating damping $\left(C_{b}\right)$, gyroscopic or centrifugal effect $(G)$, stiffness $(K)$, bearing stiffness $\left(K_{b}\right)$, centrifugal stiffness $\left(K_{c}\right)$ matrices, the force vector associated with the coordinate frame of reference $(F)$ and the frequency-dependent force vector $\left(F_{\omega}\right)$ are derived from the system components as seen in Table 1 for a rotor dynamic system at a running speed of $\Omega$. The rotating reference frame, which is not commonly used in rotor dynamic analysis, is not further discussed in this review. Table 1 shows the contributions of each component by $\otimes$, contributions sometimes modeled with $\odot$ and properties that are not typically modeled in the system with $\bigcirc$. The contributions to symmetric, skew-symmetric and unsymmetric matrices are also pointed out in Table 1.

The rotor and stator can be modeled as unsymmetric geometry and analyzed in the rotating or stationary reference frames. If the rotor and stator (support) system is unsymmetric and analyzed in the stationary reference frame, 


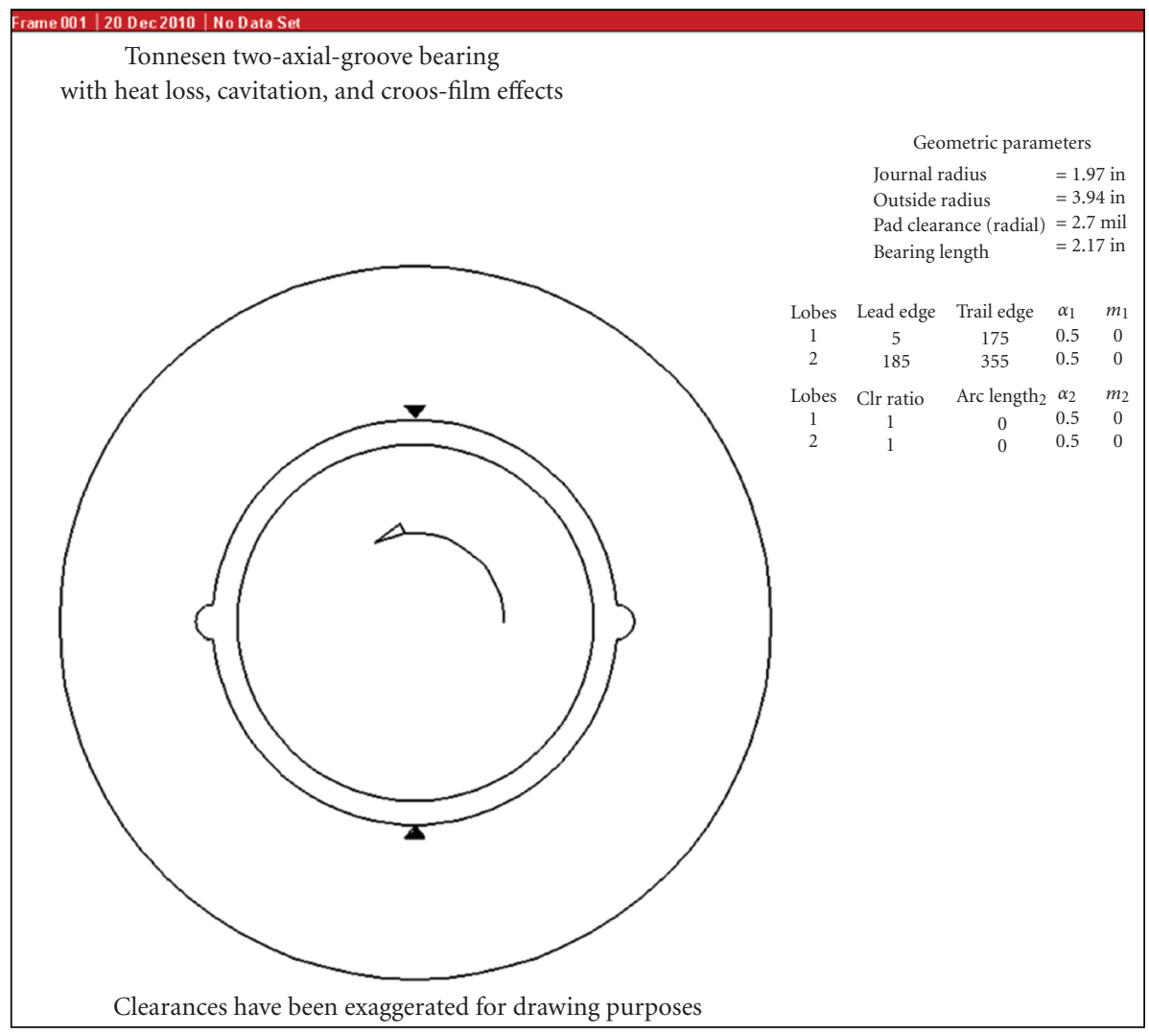

Figure 3: A two axial groove fluid film bearing.

the unsymmetric geometry of the bearings or stator will impart unsymmetric stiffness and damping on the system in the system matrices. In the rotating reference frame the unsymmetric stator or bearings are applied as external forces on the system on the right hand side of the equation. In the case of the rotating reference frame the forces applied to the rotor as an effect of the bearing and support structure are not time dependent but are harmonic and a function of the running speed. For further reference of the rotating reference frame refer to such literature as $[19,20]$.

A standard rotor dynamic analysis seeks to observe the dynamic response of the rotor system during operating conditions. A nonmathematical discussion of the rotor analyses are given by Nelson [2]. A complete but relatively simple beam type rotor dynamic analysis is discussed by Chaudhry in [1] which performs a critical speed, stability, and unbalance response analysis. Other analyses for consideration is transient analysis of specific loading scenarios for critical design for startup, failure analysis, critical speed transitions, blade loss studies, instable synchronous whirl, and nonlinear bearing analysis [21-23].

3.1. Bearing Dynamic Properties. The unsymmetric stiffness and nonproportional damping matrices incurred by the bearings arises through a typical bearing analysis. Nonproportional damping does not necessarily mean that the system damping matrix will be unsymmetric. It means that it is not proportional to a linear combination of the mass and stiffness matrices unlike proportional (Rayleigh or classical) damping.

Fluid film bearing has in general unsymmetric and nonproportional stiffness and damping matrices. Figure 3 shows a two axial groove bearing [24]. The journal bearing has a radius of $0.05 \mathrm{~m}$, length of $0.055 \mathrm{~m}$ with a radial pad clearance of $68.6 \times 10-6 \mathrm{~m}$. The bearing dynamic coefficients are calculated using thermal hydrodynamic analysis which includes the heat loss, pressure cavitation, and cross-film viscosity variation. Table 2 shows the four stiffness coefficients (Kxx, Kxy, Kyx, and Kyy) and the four damping coefficients (Cxx, Cxy, Cyx, and Cyy).

From Table 2, the coefficients are shown to be speed dependant. Moreover, the cross-coupling terms of the stiffness and damping coefficients are different which give rise to unsymmetrical matrices. The unsymmetrical behavior rises from the deviation of the steady-state operation position of the shaft from the center of the bearing. The damping coefficients matrix cannot be expressed as a linear combination of the mass and stiffness matrices.

\section{Rotor Dynamic Modeling}

A good history of rotor dynamics is provided by Allaire [25] and Nelson [26], but a brief overview will be discussed here. Rotor dynamic systems have been analyzed since Rankine, 
TABLE 2: The four stiffness and damping coefficients for the two groove axial bearing.

\begin{tabular}{lcccc}
\hline $\begin{array}{c}\text { Speed } \\
(\mathrm{rpm})\end{array}$ & $\begin{array}{c}\text { Kxx } \\
(\mathrm{N} / \mathrm{m})\end{array}$ & $\begin{array}{c}\text { Kxy } \\
(\mathrm{N} / \mathrm{m})\end{array}$ & $\begin{array}{c}\text { Kyx } \\
(\mathrm{N} / \mathrm{m})\end{array}$ & $\begin{array}{c}\text { Kyy } \\
(\mathrm{N} / \mathrm{m})\end{array}$ \\
\hline 1500 & $1.797 E+08$ & $2.285 E+07$ & $-3.739 E+08$ & $3.852 E+08$ \\
2500 & $1.617 E+08$ & $2.229 E+07$ & $-3.399 E+08$ & $3.510 E+08$ \\
3500 & $1.811 E+08$ & $6.243 E+07$ & $-3.563 E+08$ & $3.101 E+08$ \\
4500 & $1.754 E+08$ & $6.586 E+07$ & $-3.532 E+08$ & $3.071 E+08$ \\
5500 & $1.716 E+08$ & $7.013 E+07$ & $-3.540 E+08$ & $\mathrm{Cyy}$ \\
\hline Speed & $\mathrm{Cxx}$ & $\mathrm{Cxy}$ & $\mathrm{Cyx}$ & $(\mathrm{N} \cdot \mathrm{s} / \mathrm{m})$ \\
\hline$(\mathrm{rpm})$ & $(\mathrm{N} \cdot \mathrm{s} / \mathrm{m})$ & $(\mathrm{N} \cdot \mathrm{s} / \mathrm{m})$ & -1401495 & 4438158 \\
1500 & 1180944 & -1401501 & -613828 & 2370313 \\
2500 & 616542 & -613831 & -549771 & 1819237 \\
3500 & 621110 & -549773 & -382425 & 1409636 \\
4500 & 471272 & -382427 & -288805 & 1163103 \\
5500 & 383978 & -288807 & & \\
\hline
\end{tabular}

in 1869 , incorrectly determined that we cannot operate a rotor above its first critical speed. This seems to have discouraged any real development in the field for almost 50 years despite Delaval operating a single stage steam turbine above the first critical speed in the 1880's.

The first vibrational theory developed for rotor dynamic analysis was conducted by Foppl (1895) and Jeffcott (1919). Foppl mathematically showed that operation above the first critical speed is possible and stable using an undamped flexible rotor. Jeffcott added damping to the rotor system analysis. Continuing research led to the understanding and causes of rotor instabilities such as internal damping, oil whip, oil whirl, cross-coupling stiffness in bearings and seals, and gyroscopics. The Jeffcott, a single mass flexible, rotor was used by rotor dynamacs despite its simplistic formulation and assumptions to analyze complex rotor systems and effects. However, with the development and needs for analysis of more complex systems such as gas turbines and jet engines, the Jeffcott rotor would not suffice to accurately model these more complex systems, thus alternative methods were developed.

The inability and difficulty of deriving analytical models, such as the Jeffcott rotor, to model complex rotor systems led to the implementation of computational models for solving rotor dynamic systems. Developments in computing capabilities and a transition from analytical modeling to modeling the actual system geometry arose in the 1960's and 1970's. The two methods developed for such analytical modeling were the Transfer Matrix method (TMM) and the Finite Element method (FEM).

Melvin Prohl and N. Myklestad's combined efforts led to the Transfer Matrix method, still viable to this day, to handle structures having divided elements in a linear arrangement [22, 27]. This method was used for rotor modeling. It has small memory needs compared to the finite element method, but is used when the total system can be broken into a sequence of subsystems that interact only with adjacent subsystems which is not always the case in rotor systems.
In the 1970's the finite element method became available for the solution of beam-based models. Thus, rotor dynamacs began modeling the rotor systems using these beambased models via FEM for rotor analysis [28, 29]. Although there are higher computational costs associated with FEM compared with TMM, FEA "accommodates coupled behaviors of flexible disks, flexible shafts, and flexible support structures into a single massive multidimensional model" [26]. Thus, today the standard method of FEM is employed to understand more accurately the dynamic responses of rotor dynamic systems.

Modeling techniques with finite element modeling have progressed and changed since it was first utilized for rotor dynamics in the 1970's. FEM allows for complex solid modeling, complicated analysis including cross-coupling effects at rotor/disk and blade/disk interfaces, rotor-structure interactions, inclusion of nonlinear bearings, and gyroscopics. The first FEM models of rotor systems were developed via Bernoulli-Euler [30] and Timoshenko beam theory [31, 32] to take advantage of the axis symmetry of rotor. Attached components were modeled as lumped masses.

Beam elements are still used to this day as a valid method for much rotor dynamic modeling and have integrated several variations to account for more complex geometries such as hollow sections, tapered beam sections [33], modulus corrected elements for conical sections [34], and the inclusion of axisymmetric element representations of disks to a beam rotor [33]. However, with advances in computing power and some key issues with $1 \mathrm{D}$ beam models, the modeling has progressed to $2 \mathrm{D}$ axisymmetric elements and 3D solid elements as exemplified by [3539]. The main problems with beam models is that many rotor systems do not have geometry adequately modeled by $1 \mathrm{D}$ elements. The general assumption of modeling disks as lumped masses, assuming the disk is rigid, does not allow the model to account for many effects the disk imposes on the rotor system. These effects include the influence of the disk and shaft interface, complex or unsymmetric attached components, and centrifugal effects of distributed shafts, 
and the gyroscopic effects are calculated as separate elements of equivalent disks and given as inputs to the beam model [36].

Geradin and Kill [40] also point out that "models generally used (in literature) are limited to the following assumptions:

(1) the rotating and fixed parts of the structure have linear material and geometric behavior;

(2) the rotating shaft is represented by beam finite elements (with shear deformation and rotary inertia effects of right sections possibly included);

(3) the disks attached to the shaft are infinitely rigid;

(4) the stiffness and damping properties of bearings and seals are linear function of displacements and velocities."

Table 2 from [35] shows a summary of finite element methods applied to rotor systems and their capabilities, justifying the use of 3D solid finite element models. This table shows that beam-based and axisymmetric models can model specific phenomena related to gyroscopics, attached components, and varying geometry sections of rotor systems considering only symmetric geometry and components. Some research has been aimed at using axisymmetric [38], cyclic [20,41-43], and combined beam and shell models or $1 / 2$ D models [44] for analysis in an attempt to obtain smaller models. However, the axisymmetric and cyclic assumptions usually fail to model response of bladed rotor systems with unsymmetric loading scenarios due to system unbalance, fluid-structural interactions, and blade-out scenarios and cannot represent structures that do not have that specific geometry to exploit. The combined $1 / 2 \mathrm{D}$ models are not commonly used for rotor analysis in literature. Thus, to account for complex nonsymmetric systems with complex rotor geometry or nonsymmetric attached components such as bladed disks, commonly found in turbines and jet engines, modeling using 3D solid elements is needed. Solid finite elements take into account the more complex geometry, especially for larger complex structures found in many rotors.

3D solid rotor models have been used to look at complex disk geometries, attached component connections, and their response, to accurately model gyroscopics and nonlinear effects not captured in a linear formulation $[20,35,37-$ $40,48-53]$, looking at large coupled rotor-structural systems $[54,55]$, global and large scale modeling of rotating turbo machine assemblies [20], and modeling of fluid structural coupling effects of disks in rotating machines [56]. However, these models can easily reach high orders of dimensionality as seen in [50] where a bladed disk model, with no rotor or bearings, can easily reach $150000+$ DOF in a turbo machine or jet engine. There often are multiple disks and even multiple shafts, refer to the jet engine example in Figure 1, and the dimensionality can grow rapidly. The general direction of the field is towards higher speed and lower weight; this creates the need to really understand the full system dynamics [57]. Thus, the use of 3D solid models of rotor systems presents a computational problem often without adequate computing resources, leaving us with a need for a way to reduce the size of these complex systems so that they can be efficiently and accurately analyzed.

\section{Model Reduction for Rotor Dynamic Systems}

5.1. General Concepts of Model Reduction. Model reduction methods are commonly found in the fields of controls, optimization, and structural mechanics. Within these fields model reduction is often used to develop system controllers, conduct sensitivity analyses, optimize system parameters, and reduce model complexity and size for computational efficiencies $[2,4,5,13]$. In a nutshell, model reduction is a mathematical method or theory to find a low-order approximation of a large system by projecting the model onto a defined low-dimensional subspace. In the previous section, a need for model reduction of large-scale rotor dynamic models is established in order to study these complex systems.

To begin this discussion in a more general sense, it is necessary to first define what the goals are for "good" model reduction. A standard idea of these criteria is given by Noor [7] which simply states that the global approximation vectors should have:

(1) Linear independence and completeness.

(2) Low computational expense in their generation and simplicity of automatic selection of their number.

(3) Good approximation properties, in the sense of high accuracy of the solution obtained using these vectors,

(4) Simplicity of obtaining the system response characteristics using these vectors and not commonly discussed in literature.

(5) Metrics to quantify and determine the quality of reduction for dynamic analysis.

In reducing a physical system for analysis, there should be the ability to physically interpret the system, back transformations to describe the full system response, parameterization to vary specific system properties, and a way to quantify the quality of reduction for analysis.

\subsection{Considerations for Model Reduction of Rotor Dynamic} Systems. Many variations of standard methods of reduction have been developed since their initial derivation for controls systems design and structural mechanics. These methods are discussed in the previous literature reviews; refer to Section 2 for more detail. In summary, the methods from control theory typically include methods described as singular value decomposition (SVD) or methods described as Krylov methods [4]. In structural systems the methods are referred to as static and dynamic and typically include Guyan methods, real and complex modal analysis, component mode synthesis, and other formulations of modal methods [6].

Several of these methods, and modifications thereof, have been directly applied to rotor dynamic systems for both controls and dynamic analysis. However, rotor dynamics 
TABLE 3: A brief review of the capabilities of finite element methods proposed to model a rotating shaft-disk system [35].

\begin{tabular}{|c|c|c|c|c|c|c|c|c|c|c|}
\hline & \multirow[b]{2}{*}{$\begin{array}{l}\text { Rotary } \\
\text { inertia }\end{array}$} & \multirow[b]{2}{*}{$\begin{array}{l}\text { Gyro- } \\
\text { scopic } \\
\text { effect }\end{array}$} & \multirow[b]{2}{*}{$\begin{array}{c}\text { Shear } \\
\text { deformation }\end{array}$} & \multicolumn{2}{|c|}{$\begin{array}{l}\text { Prismatic solid or hollow } \\
\text { cross-section }\end{array}$} & \multirow[b]{2}{*}{$\begin{array}{l}\text { Solid } \\
\text { axisym- } \\
\text { metric }\end{array}$} & \multirow{2}{*}{$\begin{array}{l}\text { Tapered } \\
\text { Two } \\
\text { principle } \\
\text { axis } \\
\end{array}$} & \multirow[b]{2}{*}{$\begin{array}{l}\text { Hollow } \\
\text { axisym- } \\
\text { metric }\end{array}$} & \multicolumn{2}{|c|}{$\begin{array}{l}\text { Abrupt change } \\
\text { in cross-section }\end{array}$} \\
\hline & & & & $\begin{array}{l}\text { Axisym- } \\
\text { metric }\end{array}$ & $\begin{array}{c}\text { Two } \\
\text { principal } \\
\text { axes }\end{array}$ & & & & $\begin{array}{l}\text { Axisym- } \\
\text { metric }\end{array}$ & $\begin{array}{c}\text { Non } \\
\text { axisym- } \\
\text { metric }\end{array}$ \\
\hline $\begin{array}{l}\text { Rayleigh } \\
\text { Shaft element } \\
{[28]}\end{array}$ & $\checkmark$ & $\checkmark$ & $\mathrm{x}$ & $\checkmark$ & $\checkmark$ & $\mathrm{x}$ & $\mathrm{x}$ & $\mathrm{x}$ & $\mathrm{x}$ & $\mathrm{x}$ \\
\hline $\begin{array}{l}\text { Conical shaft } \\
\text { element } \\
\text { based on } \\
\text { Timoshenko } \\
\text { beam } \\
{[17,31,45-} \\
47]\end{array}$ & $\checkmark$ & $\checkmark$ & $\checkmark$ & $\checkmark$ & $\mathrm{x}$ & $\checkmark$ & $\mathrm{x}$ & $\checkmark$ & $\mathrm{x}$ & $\mathrm{x}$ \\
\hline $\begin{array}{l}\text { Modulus } \\
\text { corrected } \\
\text { elements [34] }\end{array}$ & $\checkmark$ & $\checkmark$ & $\checkmark$ & $\checkmark$ & $\checkmark$ & $\checkmark$ & $\checkmark$ & $\checkmark$ & $\checkmark$ & $\mathrm{x}$ \\
\hline $\begin{array}{l}\text { Axi- } \\
\text { symmetric } \\
\text { finite } \\
\text { elements } \\
{[38,48]}\end{array}$ & $\checkmark$ & $\checkmark$ & $\checkmark$ & $\checkmark$ & $\mathrm{x}$ & $\checkmark$ & $\mathrm{x}$ & $\checkmark$ & $\checkmark$ & $\mathrm{x}$ \\
\hline $\begin{array}{l}\text { Three- } \\
\text { dimensional } \\
\text { solid finite } \\
\text { element [37] }\end{array}$ & $\checkmark$ & $\checkmark$ & $\checkmark$ & $\checkmark$ & $\checkmark$ & $\checkmark$ & $\checkmark$ & $\checkmark$ & $\checkmark$ & $\checkmark$ \\
\hline
\end{tabular}

have specific considerations in the physical structure of the system, and as a result present mathematical and computational difficulties are not found in most control or structural models for which these methods were developed. The main difficulties and differences presented in rotor dynamic systems are the inclusion of nonsymmetric stiffness, nonproportional damping, and gyroscopics effects, see Table 3.

The nonsymmetric stiffness and nonproportional damping, normally arising from the bearings, impart unsymmetric matrices in the system dynamic equations of motion as shown in the previous Section 3. The gyroscopics, inherent in rotating systems, impart a skew-symmetric matrix of cross-coupling terms that are also frequency, or running speed, dependent. The speed-dependent properties equate to modified system matrices for each speed that the system is analyzed for and requires consideration for the reduction basis at each sunning speed. The concentrated damping forces result from bearings and other support structures in the system and are nonproportional, can be nonlinear and can be frequency dependent.

These structural complexities pose three potential problems for each model reduction method and system analysis: (1) unsymmetric and skew-symmetric matrices, (2) speed-dependent properties, and (3) sparse and possibly ill-conditioned matrices. The speed-dependent properties impart an important consideration in model reduction can an efficient basis for reduction be developed independent of the running speed or is a basis that is a function of running speed necessary to reduce the system for analysis. Since most methods for reducing models come from methods developed for controls and structural analysis, for symmetric system matrices, a careful understanding needs to be developed to determine the best methods to reduce rotor dynamic systems.

5.3. Applications of Model Reduction for Rotor Dynamic Systems. The field of rotor dynamics has implemented model reduction methods in an attempt to address the high dimensionality of the systems and at times modeled unsymmetric stiffness, gyroscopics, and nonproportional damping. As previously mentioned, the methods used for rotor dynamic systems are native to both controls and structural reduction. From an extensive survey of the literature, the most common methods used for structural reduction of rotor systems appear to be Guyan Reduction [38, 58], Modal Analysis [39, 59-63] and Component Mode Synthesis [20, 40, 43, 64-66]. More specifically research on reduction methods, not necessarily applied to rotor systems, looks to handle damping [59, 65, 67-69] and gyroscopics [63, 70-72] within the systems but rarely together. Other methods such as Krylov-based methods have been applied to structural systems for FEA analysis [73] but are not commonly found in the literature nor are they normally employed in rotor systems. Another small subclass of reduction not as common to rotor dynamics, Parametric Model Order Reduction 
as presented by Baur et al. [74], Panzer et al. [75], and Daniel et al. [76] for nonrotor dynamic applications and by Ganine [77] for modeling of misaligned stacked disks rotor assemblies, is based on the orthogonal projection of system equations to Fourier basis vectors corresponding to lower harmonics of the system.

Common reduction methods found for developing controllers for rotor dynamic systems are balanced truncation/realization [11, 78-81], Structure preserving transformations (SPT) [59], System Equivalent Reduction Expansion Process (SEREP), and modified SEREP [5]. Krylov-based methods as found in $[82,83]$ are used for controller development of nonrotating systems. The Krylov-based methods are not normally employed in rotor systems and will not be discussed in further detail in this review. It is also interesting to point out that the BT, SPT, and SEREP methods are based on real and complex eigenvector modal methods that attempt to preserve the system dynamics through reduction.

Research by Tasaltin concludes that the modal and internal balancing methods are the most suitable reduction methods for rotor dynamic systems [84]. Thus, from the literature it can be determined that the most common methods applied to rotor systems are Guyan, MA, CMS, and BT methods with the most common basis being that of the eigenvectors (a modal basis).

5.3.1. Guyan Reduction. Guyan reduction, also known as static reduction, reduces the system matrices of a system by dividing the coordinate into master and slave coordinates $[6,85,86]$. The slave coordinates are assumed to have low inertia relative to stiffness and are constrained to displace as dictated by elastic properties and displacements associated with other coordinates defined as the masters. For undamped free vibration the DOF can be associated with either master $(m)$ or slave $(s)$ :

$$
\left[\left[\begin{array}{cc}
K_{m m} & K_{m s} \\
K_{m s} & K_{s s}
\end{array}\right]-\omega^{2}\left[\begin{array}{cc}
M_{m m} & M_{m s} \\
M_{m s} & M_{s s}
\end{array}\right]\right]\left\{\begin{array}{l}
\bar{D}_{m} \\
\bar{D}_{s}
\end{array}\right\}=\left\{\begin{array}{l}
0 \\
0
\end{array}\right\},
$$

of the partitioned stiffness and mass matrices. From these partitioned matrices it is possible to relate the slave and master DOF of the stiffness matrices through a transformation:

$$
\left[T_{r}\right]=\left[\begin{array}{c}
I \\
-K_{s s}^{-1} K_{m s}^{T}
\end{array}\right]
$$

From this we can generalize and write the general equations of motion for a system as

$$
\left[M_{r}\right] \ddot{D}_{m}+\left[C_{r}\right] \dot{D}_{m}+\left[K_{r}\right] D_{m}=\left[F_{r}\right],
$$

where

$$
\begin{array}{cc}
{\left[M_{r}=T_{r}^{T} M T_{r}\right],} & {\left[C_{r}=T_{r}^{T} C T_{r}\right],} \\
{\left[K_{r}=T_{r}^{T} K T_{r}\right],} & {\left[F_{r}=T_{r}^{T} F\right] .}
\end{array}
$$

The master DOF would typically consist of lumped disks, external force locations, imbalance locations, bearing locations, and so forth. This reduces a system to only the defined master coordinates $(m)$ from the total coordinates $(m+s)$ and as described can only approximately represent the secondorder system dynamics because it assumes that the dynamic relationship between retained and discarded DOF is same as the static relationship. Also, the system is not uncoupled but can be used for stability, synchronous response, and transient analysis. Note that although Guyan reduction is commonly found throughout the literature it generally does not reduce systems well for dynamic analysis: "Using classical reduction techniques, such as Guyan reduction, it is possible to reduce the size of the matrices, but at the expense of poor model accuracy [87]." This is further discussed in the literature.

Rouch and Kao [58] presented the use of Guyan reduction for rotor dynamic systems for critical speed, stability, forced response, and transient analysis. A small order finite element model, of 45 elements with 4 DOF at each node, was reduced by Guyan reduction for the analysis and comparison to that of experimental data for critical speed and stability analysis. Stephenson and Rouch [38] used Guyan reduction with axisymmetric harmonic elements to reduce rotor models, including system gyroscopics. The reduced model by Guyan reduction, with master DOF selected based upon beam bending behavior, showed poor correlation to the experimental results. They performed a brief investigation of the effects of master DOF on simple beam models but emphasize that the selection of master degrees of freedom is determined by the analyst. Houlston et al. [59] used Guyan reduction to reduce rotor-disc models before applying their proposed structure preserving method (SPT).

5.3.2. Modal Analysis. Modal analysis [6, 23, 88] uses modal coordinates to represent the system and decouple the dynamic system equations of motion. The modes of the system are calculated via an eigenvalue analysis where the number of modes equals the number of DOF in the system. Typically higher order modes above about twice the operating frequency are discarded to reduce the system size [6]. The selection and retention of modes is another area of research within the methods for basis selection. The reduction in the system comes from a retained subset of the system modes $(m)$ from the full set of modes.

Real modal analysis uses real undamped eigenvalues easily calculated from the $M$ and $K$ system matrices from the standard eigenvalue problem

$$
\left[\lambda I-\left[M^{-1} K\right]\right] u=0 .
$$

The eigenvectors are typically mass normalized so that the reduced mass matrix is identity, (8), and the reduced stiffness matrix becomes the square of the real eigenvalues $\omega^{2},(9)$. A modal matrix $[\Phi]$ whose columns are the eigenvectors normalized with respect to the mass matrix, provide a reduced basis from the $n$ original natural frequencies in the system. The reduction of the system mass and stiffness matrices can be seen in (8) and (9).

$$
\begin{gathered}
{[\Phi]^{T}[M][\Phi]=[I],} \\
{[\Phi]^{T}[K][\Phi]=\left[\omega^{2}\right],}
\end{gathered}
$$


where the reduced matrices $[I]$ and $\left[\omega^{2}\right]$ are diagonal and dimension $m \times m$, where $m$ is the number of modes retained for analysis.

Complex modal analysis uses damping, gyroscopic, or both system matrices to yield complex conjugate pairs of eigenvectors, referred to as left and right eigenvectors, used for the system reduction calculated from the generalized eigenvalue problem in state space representation. The formulation for complex modal analysis is readily discussed by Khulief and Younan [61, 89].

The system is written in state space formulation as:

$$
[A] \dot{q}+[B] q=0,
$$

where

$$
\begin{gathered}
A=\left[\begin{array}{cc}
0 & M \\
M & C+\Omega G
\end{array}\right], \\
B=\left[\begin{array}{cc}
-M & 0 \\
0 & K
\end{array}\right], \\
q=\left[\begin{array}{l}
\dot{u} \\
u
\end{array}\right],
\end{gathered}
$$

are the nodal displacement and velocities. The right eigenvectors and natural frequencies are obtained from

$$
[\lambda A+B] \Phi_{R}=0,
$$

where $\lambda$ are the complex eigenvalues and $\Phi_{R}$ are the corresponding right eigenvectors. The left eigenvectors are obtained in the same manner from the equation:

$$
[\lambda A+B]^{T} \Phi_{L}=0,
$$

where $\lambda$ are the complex eigenvalues and $\Phi_{L}$ are the corresponding left eigenvectors. The left and right eigenvectors are then typically normalized so that the system matrices are then:

$$
\begin{gathered}
{\left[\Phi_{L}\right]^{T}[A]\left[\Phi_{R}\right]=[I],} \\
{\left[\Phi_{L}\right]^{T}[B]\left[\Phi_{R}\right]=\left[\omega^{2}\right],}
\end{gathered}
$$

where the system matrices $A$ and $B$ are reduced matrices to the identity $[I]$ and square of the natural frequency $\left[\omega^{2}\right]$, respectively. These reduced matrices are diagonal and dimension $2 m \times 2 m$, where $m$ is the number of complex modes retained for analysis.

Complex modal analysis is not as commonly used because of its computational complexity from retention of the damping and very importantly the frequency-dependent gyroscopics in the eigenanalysis. Through this reduction the state matrices, $A$ and $B$, are decoupled from one another, whereas in the real analysis the $M$ and $K$ matrices are decoupled. If the unsymmetric stiffness included in the analysis, nonproportional damping or gyroscopics are usually not decoupled using real modal analysis, unless retaining only the diagonal terms and assuming the offdiagonal terms are negligible following the transformations through reduction. Usually this is a poor assumption for rotor systems.

There are several publications in the literature that used modal analysis as a solution method for dynamic analysis, not model reduction. Laurenson [90] discussed modal analysis and the influence of the structure and spin speed on the dynamics of flexible rotating structures. Lee et al. [62] used modal analysis as a solution method to investigate anisotropic and isotropic boundary conditions on a continuous Rayleigh shaft without reduction of the system. Hong and Park [91] used complex modal analysis without truncation as a solution method, not for reduction, of a distributed parameter rotor bearing system using exact dynamic elements. The proposed method used a global system matrix derived from Timoshenko beam theory in the Laplace domain and accounted for the anisotropy in rotor-bearing systems by using complex conjugate partial differential equations. This method is successfully applied to a 7-node shaft with varying cross-sections and isotropic bearings. Jei and Lee [60] developed a modal analysis solution method for a rotating uniform asymmetrical Rayleigh shaft rotor system that has asymmetrical rigid disks and isotropic bearings. They then evaluate the effects of boundary conditions and rotor asymmetry on modal properties and stabilities of the system.

Much of the literature is limited in its scope and application of unsymmetric stiffness, damped systems, and gyroscopics. Wang and Kirkhope [63] presented an eigensolution method for the calculation of whirl frequencies and critical speeds of systems with gyroscopics and no other crosscoupling effects or damping. This method utilizes the system matrices, but is restricted to systems without damping. It uses real eigenvectors to decouple modal equations. Nandi [19] used complex modal analysis to reduce a nonaxisymmetric rotor on nonisotropic spring support, no damping, in a rotating frame. This work is an initial step to more complex rotor dynamic systems with damping, complex geometries and $3 \mathrm{~d}$ solid models. Mahadevan et al. [92] considered the synchronous whirl of axisymmetric rotors on rigid bearings with the gyroscopics implicitly described in the system equations using modal projections of the systems nonspinning vibration mode shapes. The main assumption presented in the paper for modal analysis, using nonspinning mode shapes, is that the gyroscopic terms can be viewed as arising from a state of prestress from a nonzero spin rate in a direct continuum mechanics representation. Mahadevan estimated and accurately compared the critical speeds on arbitrary beam-plus-rigid body axisymmetric rotor models using the modal projection methods.

Other authors sought to discern the differences between real and complex modal analysis, and how these formulations handle lightly or nonclassically damped rotor dynamic systems with the inclusion of gyroscopic system. Khulief and Mohiuddin [61] provided a comparison of real and complex modal analysis for a rotor-bearing system with anisotropic bearings and gyroscopics for a dynamic analysis. They conclude that there is little difference between the accuracy with 
the use of planar and complex modes for reduction on simple beam finite element models, comparing planar reduced models to the complex natural frequencies of the full system, not a reduced system via complex modes. Choy and Gunter [93] studied the effects of real and complex modal analysis on beam-based models of rotor dynamic systems and the effects on the critical speed, stability, unbalance response and transient analysis. Choy emphasize the importance of including the nonproportional damping from the bearings, presents a method to approximate damped critical speeds from undamped critical speeds, and concludes that both undamped and damped modal methods can be used for the analysis. A following study by Gunter and Choy [94] presented the theory of modal analysis for rotor dynamic analysis using undamped modal analysis coupling the modal equations by the generalized bearing coefficients and gyroscopic moments. This method allows for rapid analysis of a rotor but an analysis using only undamped critical speeds or decoupled modal analysis assuming proportional damping may lead to erroneous results when considering unsymmetric bearings, seals, or hydrodynamic effects in the system.

Other literature used modal analysis as a secondary reduction method. Houlston et al. [59] used structure preserving transformations via Lancaster Augmented matrices to diagonalize a nonclassically damped system and preserve second-order dynamics to develop a modal controller.

5.3.3. Component Mode Synthesis. Component mode synthesis $[6,86,95,96]$ is a method by which a system is divided into smaller structures that are individually designed, analyzed, and then assembled into a full system model for a dynamic analysis. For fixed CMS each subsystem is analyzed for its component modes: vibration modes with the substructure interface DOF fixed and constraint modes: static displacement patterns produced by applying a unit displacement to each interface DOF in turn, while all others are kept fixed. Thus the system is reduced by retaining a subset of the modes for each subsystem and by the number of interface DOF between the systems. The system is represented by a combination of physical and modal coordinates. The complete structure in reduced form is "synthesized" by assembling substructures along shared interface nodes. One disadvantage of CMS is that the final equations are not normally uncoupled. However, this reduction does contain the full system dynamics from modal analysis and the modal representation are independent of the boundary and interface coordinates which are attractive for modeling nonlinear supports such as bearings. A detailed mathematical formulation of CMS can be found in Craig and Bampton [96] or Qu [6].

Several variations of component mode synthesis have been developed since the development of the fixed interface method [95, 96]. Apiwattanalunggarn et al. [97] developed a nonlinear extension of the fixed interface method, using nonlinear normal modes for application to large-scale structural systems based on finite element methods. Variations of the CMS method also greatly vary because of the variations of boundary conditions imposed on the substructures during analysis and consequently how the assembled structure is put together. Biondi and Muscolino [98] explored the boundary conditions of the substructures, considering them as fixedfixed, fixed-free, free-free, and fixed with overlapping elements for the primary and secondary substructures fixed. With comparisons to traditional modal analysis it was shown that these different interface methods can represent the system dynamics with that of the free-fixed interface method being the most accurate.

Much of the published literature seeks to study the effects of attached components on a rotor dynamic system that cannot inherently be captured through beam-based models. Chatelet et al. [20] analyzed a one-dimensional beam model of a flexible bladed disc-shaft assembly including centrifugal stiffening and gyroscopics for analysis of the rotating system. Chatelet used the nonrotating system modes $(\Omega=0)$ to reduce the system using the Craig-Bampton method [96]. This study shows that the system dynamics may be poorly modeled using tradition modeling techniques that are based on different uncoupled models. Bladh $[49,50]$ formulated the Craig and Bampton CMS method with a secondary modal analysis for application to mistuned bladed disks showing significant reduction in computational costs and good accuracy. However, these bladed disk models that include only structural damping are nonrotating and are not analyzed with a full rotor-dynamic system. Bauchau et al. [54] modeled a coupled rotor-fuselage undergoing large maneuver angles using Herting's transformation as opposed to the Craig-Bampton method. This analysis was done to study the coupling of a fuselage and rotor system in one dynamic analysis for transient analysis of maneuvers and was found to show good agreement; despite of the level of complexity, the rotor system is unclear.

The other main focus of the literature on component mode synthesis applied to rotor dynamic systems is the study of the boundary conditions used for the system assembly. Shanmugam and Padmanabhan [64] presented a hybrid fixed-free interface method for rotor analysis of a $1 \mathrm{~d}$ beam model including gyroscopic effects and symmetric damping to predict the whirl frequencies and unbalance response. They apply this method to a simple cantilever rotor with a heavy disk at the free end representing a typical twin spool engine model. Using component modes, assuming that the gyroscopics do not significantly change with speed, they reduce the system model and accurately predict the rotor response with the hybrid fixed-free method, comparable to results of the Craig-Bampton method, compared to the full model. Tran [42] presented a method using partial interface modes and applied the method to tuned and mistuned structures such as bladed disks with cyclic symmetry. The partial interface method uses the normal modes resulting from the static condensation of the structure to the interface of subsequent substructures. Tran shows good correlation between the reduced models with partial interface CMS to their reference model. Wang and Kirkhope [65] presented a free interface CMS method using real modes, residual flexibility, inertia relief modes, and residual dissipative effects to model a damped rotor system with either rigid or flexible interfaces. They show good correlation between the reduced models to a $176 \mathrm{DOF}$ beam model of a two-spool aircraft 
turbine engine to predict whirl frequencies and damping exponents.

Other studies seek to study the effects of truncation through the CMS method and the effects on a rotor dynamic analysis. Li and Gunter [99] performed a critical speed, stability and forced response analysis on the beam-based model for generally connected multicomponent systems. Li's study of undamped modal truncation error in CMS on a dual rotor-system engine suggests that selection of modes is based on an upper frequency limit 4 to 5 times that of the top rotor speed and cautions the interpretation of the calculation of the damping and unbalance response which show no consistent trend in error like the overestimation of the natural frequencies. Subbiah et al. [100] used component mode methods applied to rotor dynamic problems considering only the lower modes of the system to reduce the system matrices to perform an unbalance response of a simple Jeffcott rotor, no gyroscopics, compared with experimental work. Iwatsubo et al. [101] presented a method for including nonlinear vibrational analysis for a rotor system using CMS and harmonic balancing methods. However, the equations of motion are explicitly derived for each frequency and component and assembled using CMS.

5.3.4. Balanced Truncation. Balanced Truncation (realization), as developed by Moore [102] and discussed by Burl [103], is the transformation of the system states so that observability and controllability grammians of the transformed system are equal and diagonal. This is based on the use of state-space realization, (15), where it is assumed that each state is equally strongly coupled to the input and the output. This allows an ordering of the states based on their input-output coupling; reduced order systems can then be created by truncating states that weakly participate in the input-output behavior. Quantification of the coupling of states to the input and output can be done by using the controllability and observability grammians, respectively. However, it may be difficult or numerically inefficient to develop a transformation that provides the balanced system and equate the observability and controllability grammians [57].

Considering generic state space model that is linear time invariant

$$
\begin{aligned}
& \dot{x}(t)=A x(t)+B u(t), \\
& y(t)=C x(t)+D u(t) .
\end{aligned}
$$

For a system with $p$ inputs, $q$ outputs and $n$ states, $x(t)$ is the state of the system, $y(t)$ is the output of the system, $u(t)$ is the input (control vector) of the system, $A$ is the state matrix $(n \times n), B$ is the input matrix $(n \times p), C$ is the output matrix $(q \times n)$ and $D$ is the feedthrough matrix $(q \times p)$. The state space system can be represented in physical coordinates, modal coordinates, or some other basis before the system is balanced for reduction.

The controllability Grammian is

$$
L_{c}=\int_{0}^{\infty} e^{A t} B B^{T} e^{A^{T} t} d t
$$

and can be computed by solving the Lyapunov equation:

$$
A L_{c}+L_{c} A^{T}+B B^{T}=0 .
$$

The observability Grammian is

$$
L_{o}=\int_{0}^{\infty} e^{A^{T} t} C C^{T} e^{A t} d t
$$

and can be computed by solving the Lyapunov equation

$$
A^{T} L_{o}+L_{o} A+C^{T} C=0 .
$$

In order to generate a balanced realization a similarity transformation on the original state space system is needed and as an example of this is selected as follows from Burl [103], where in this formulation the balancing is not guaranteed to be unique or the numerically optimal one.

(i) Factor the original controllability grammian: $L_{c}=$ $R_{c} R c^{T}$ where the $R$ matrix is positive definite.

(ii) Compute $R c^{T} L_{o} R_{c}$.

(iii) Solve for the eigenvalues and eigenvectors of matrix $R c^{T} L_{o} R_{c}: R c^{T} L_{o} R_{c} U_{c o}=U_{c o} \Lambda_{c}$.

(iv) Compute the balanced transformation matrix $T_{b}=$ $L_{c} U_{c o} \Lambda^{1 / 2}$.

Thus, by using this transformation on the state space matrices the grammians can be made equal, or balanced:

$$
L_{o}=L_{c}=\left(\begin{array}{ccc}
\sigma_{1} & & 0 \\
& \ddots & \\
& & \sigma_{n}
\end{array}\right) \text {, }
$$

where the diagonal elements are real, positive, and ordered from largest to smallest for convention, and each element correspond to a single state. The resulting states can be ordered in some manner such as the Hankel norm to determine which contribute the most to the system dynamics.

Examples of the BT methods applied to rotor dynamic systems are primarily focused on controller design for the rotor systems. These controller designs are typically for specific rotor systems with light damping and gyroscopics that make balancing the system feasible. Some examples in the literature are discussed below. However, one main feature of rotor dynamic analysis is the quantification of the stability of unstable systems. This inherently renders the Balanced Truncation approach unlikely useful for rotor dynamic purposes as the computation of a balanced realization of the system matrices requires that the system is stable and minimal.

Sawicki and Gawronski [78] implemented balanced truncation in the development of a linear quadratic controller for the control of vibration of a flexible rotor bearing system with small gyroscopic effects. The reduction is done in two steps using balanced truncation and then a LQR reduction using a condition that allows one to ignore the gyroscopic effects in the controller design but that are included in the rotor dynamics. 
Mohiuddin et al. [57] applied the direct truncation balanced model reduction method to a complex rotor bearing system with gyroscopics, rotary inertia, and light damping for analysis and controller design. The authors presented the use of the singular perturbation balanced truncation scheme to alleviate the steady-state offset error common to balanced truncation. The authors also applied complex modal analysis to reduce a complex model before applying the presented balanced reduction methods.

Fan et al. [79] used the complex modes and balanced realization methods to design an LQ regulator that can accurately control a rotor system with light damping and gyroscopics within a defined frequency range. The authors used the normal (planar) modes of the system, as opposed to the processional (gyroscopic influenced) modes, to reduce the model and develop the controller. The authors showed that the modal method provides greater model accuracy over the balanced realization method. It was also shown that the balanced model reduction method for a defined frequency range of a high order lightly damped mechanical systems results in poorly correlation to the actual system because the approximation originates from the entire frequency range of the system.

5.3.5. Other Reduction Methods. Das and Dutt [5] presented a modified Structure Equivalent Reduction Expansion Process (SEREP) method based upon the use of complex modal analysis and selection by modal amplification factor for systems with gyroscopics, internal and external damping. The second step of the reduction is to classify the states in terms of active and deleted states, upon which the deleted states are related to the active states via Guyan reduction; 'there is no criterion of selecting the active states except for predicting a good response and reducing the order of the system. Their method goes on to show that the reduced order model for a $56 \mathrm{DOF}$ system can accurately predict the system response up to the frequency for which the mode was retained.

5.3.6. A Summary of the State of Reduction Methods Applied to Rotor Dynamic Systems. Reduction methods have been applied to rotor dynamic system. A close look at some of the literature provides several deficiencies when using some of these methods or for applications to rotor systems. The common theme in the literature is (1) to present new variations of methods, (2) to apply the reduction methods to small finite element models that do not necessarily need reduction, and (3) to make several assumptions to reduce the dimensionality of the system or eliminate or mitigate the rotor dynamic effects before the reduction method presented is used. No literature has shown a large-scale rotor dynamic analysis and the effects of various model reduction methods on the analysis. To give a brief example of these issues from the discussed literature, we give the following.

(1) Bladh $[49,50]$ presented solid finite element models of bladed disks with structural damping, nonrotating, no gyroscopics and performed a secondary reduction method following component mode synthesis.

(2) Das and Dutt [5] presented a modified SEREP method, based on complex modal analysis, that includes internal and external damping along with gyroscopics, but their presented example is for a beam model of a rotor where they reduce the order of the model by only preserving translational DOF, the gyroscopics couple translation, and rotational DOF.

(3) Wang and Kirkhope $[65,69]$ propose a CMS method for damped rotor systems and again apply it to a complex system modeled with beam elements but not accounting for gyroscopics.

(4) Houlston et al. [59] presented an SPT method for nonproportional damping of rotating machines where the system is reduced via Lancaster Augmented matrices and then diagonalized (decoupled) via complex modal analysis. Houlston does not include gyroscopics or apply their method to a complex model; in fact they use Guyan reduction on a simple example before applying their method.

(5) Mohiuddin et al. [57] studied balanced truncation applied to a rotor system with anisotropic bearings, gyroscopics, and shear but again present a very simplified model for their reduction.

(6) Wang and Kirkhope [69] presented results for modal analysis on a simple slightly damped rotor with no cross-coupling except gyroscopics.

(7) Bauchau et al. [54] couple rotor-foundations for analysis via a method of CMS which focuses on the coupling of systems and not the inclusion of rotor characteristics.

(8) Fan et al. [79] used a variation of balanced truncation, but due to frequency-dependent effects not accounted for, cannot accurately analyze the whole frequency range.

These examples are repeatedly found through the literature and make it difficult to ascertain what reduction methods work well and how they compare to one another in handling the reduction of rotor systems.

\section{Model Reduction Metrics}

Model reduction metrics serve the purpose of defining and creating a reduced model that meets the criteria discussed in the previous section. The main idea is that a reduced model, with a significant order of reduction, can be used to accurately analyze a rotor dynamic system. This depends not only on the reduction method used, described in the previous section, but also on how the basis of reduction is chosen and following reduction the ability to say "we have a good reduced model." Thus, metrics are needed in order to determine if the reduced model is adequate without ever analyzing the full scale models, the whole reason for reduction. 
Before a system is reduced, the basis for reduction is selected with some a priori knowledge that allows the user to pick an approximate space to reduce the model with. In Guyan reduction selecting masters and slave DOF is typically based on user experience and with an understanding of the mode shapes and key points of interest for loading, bearings, and so on $[6,58,86]$. In modal analysis the selection of the modes to retain can include, up to the mode of interest, twice the frequency of interest $[5,104]$, using a middle frequency range [105] and more specifically for rotor dynamics using synchronous modes [1]. In CMS the selection of both interface DOF, the discretization of the system, and the modes of each subsystem to retain are determined by the user and follow similar selection to that of modal analysis. However, in CMS there are many methods that use fixedinterface, free-interface, and hybrid interface methods as discussed in $[41,64,65,98]$. In balanced truncation the states (modes) that are most observable and controllable are used to form the reduced basis of the model by selection by an error criterion via the infinity norm or a frequency weighted infinity norm $[11,78,79]$ or energy in the state by the Hankel singular value expresses the amount of energy in each state.

It is apparent that there should be a criteria for selecting a reduced basis and with the intention of minimizing the error of the reduced model output to that of the full model output. The literature often uses low-order beam-based models, with typically less than $100 \mathrm{DOF}$ that can easily be analyzed without reduction, to show how reduction affects the accuracy of the model. Even when this error can be quantified it is not readily reported in the literature. When reducing a large order model it might not be feasible to compare computational results of the reduced model to that of the full model to ensure a good approximation of the system.

Some proposed measures that could be used for the quantification of the reduction on the system and its dynamic similarity to the full order model are discussed. These metrics include the percent error of natural frequency, correlated coefficient for modal vector, the Hankel norm, and a relative error for system output. Of these metrics the first is the only typically seen in the literature. The percent error of natural frequency:

$$
\operatorname{PE}\left(\omega_{j}^{i}\right)=\frac{\omega_{j}^{i}-\omega_{j}}{\omega_{j}} 100 \%,
$$

where $\omega_{j}$ and $\omega_{j}^{i}$ are the exact and $i$ th approximation of the $j$ th frequency. The correlated coefficient for modal vector (CCFMV) value presented by $\mathrm{Qu}[6]$ is defined

$$
\operatorname{CCFMV}\left(\phi_{m j}^{i}\right)=\frac{\phi_{m j}^{T} \phi_{m j}^{i}}{\left(\left[\phi_{m j}^{T} \phi_{m j}^{i}\right]\left[\left(\phi_{m j}^{i}\right)^{T} \phi_{m j}^{i}\right]\right)^{0.5}},
$$

where $\phi_{m j}$ and $\phi_{m j}^{i}$ are the exact and ith approximation of the $\mathrm{jth}$ eigenvector. A value of CCFMV close to 1 means that the two eigenvectors are well correlated. The Hankel norm could be used to compare the energy represented in the relative states or modes of the system. The relative error between reduced models could be used to reach a threshold and determine an appropriate basis for reduction as commonly used in FEM to determine sufficient discretization of elements within a model.

\section{Future Direction of Work and Concluding Remarks}

Previous reviews discuss model reduction applied to structural and control problems where the systems are typically selfadjoint and symmetric, for which these methods were derived. The literature also applies these reduction methods to rotor dynamic systems for analysis. The common methods seen applied to rotor dynamic systems are Guyan reduction, real and complex modal analysis, component mode synthesis, and balanced truncation.

Although many model reduction methods have already been applied to rotor dynamic systems, there is still a gap in the understanding and application of these reduction methods to rotor dynamic systems. The application of these methods typically does not address the common properties inherent to rotor dynamic systems: unsymmetric stiffness, nonproportional damping, or gyroscopics. The examples that the literature uses are small in their computational size where reduction is not necessary for analysis. The literature does not extensively compare these model reduction methods applied to a realistic rotor dynamic system that would be analyzed in industry. Nor does the literature use a large order complex model for a complete rotor dynamic analysis that would have significant gyroscopic effects, unsymmetric stiffness, and nonproportional damping from the bearings.

Future work to clarify and improve the application of the common reduction methods to rotor dynamic systems should answer

(1) how these different methods seek to handle rotor dynamic systems,

(2) how each of these methods reduce a full rotor dynamic system for a complete rotor dynamic analysis,

(3) how these methods compare to one another in reduction and modeling capabilities,

(4) what basis should be used to reduce the system,

(5) what reduction metrics determine a good reduced rotor dynamic system.

A comparison of methods used in the literature on a large order rotor dynamic system with unsymmetric stiffness, nonproportional damping, and gyroscopics can answer most of these questions.

\section{References}

[1] J. Chaudhry, M. Wagner, F. He et al. et al., "A complete rotordynamic analysis of a rotor-disk system using finite element method," in Proceedings of the 8th IFToMM International Conference on Rotordynamics, KIST, Seoul, Korea, September 2010 . 
[2] F. C. Nelson, "Rotor dynamics without equations," International Journal of Condition Monitoring and Diagnostic Engineering Management, vol. 10, no. 3, pp. 2-10, 2007.

[3] J. Chaudhry, T. Dimond, A. Younan, and P. Allaire, "Lateral rotordynamic analysis of a large alternator/flywheel/motor train," in Proceedings of the ASME Turbo Expo, June 2010.

[4] A. C. Antoulas, D. C. Sorensen, and S. Gugercin, "A survey of model reduction methods for large-scale systems," Contemporary Mathematics, vol. 280, pp. 193-219, 2001.

[5] A. S. Das and J. K. Dutt, "Reduced model of a rotorshaft system using modified SEREP," Mechanics Research Communications, vol. 35, no. 6, pp. 398-407, 2008.

[6] Z. Qu, Model Order Reduction Techniques, Springer, New York, NY, USA, 2004.

[7] A. K. Noor, "Recent advances and applications of reduction methods," Applied Mechanics Reviews, vol. 47, no. 5, pp. 125146, 1994.

[8] S. Gugercin and A. C. Antoulas, "A survey of model reduction by balanced truncation and some new results," International Journal of Control, vol. 77, no. 8, pp. 748-766, 2004.

[9] A. C. Antoulas, Approximation of Large-Scale Dynamical Systems, Society for Industrial and Applied Mathematics, 2005.

[10] A. Ghafoor and V. Sreeram, "Frequency weighted balanced model reduction: a survey," in Proceedings of the 9th International Conference on Control, Automation, Robotics and Vision (ICARCV'06), pp. 1-6, December 2006.

[11] D. F. Enns, "Model reduction with balanced realizations: an error bound and a frequency weighted generalization," in Proceedings of the 23rd IEEE Conference on Decision and Control, pp. 127-132, December 1984.

[12] C. A. Lin and T. Y. Chiu, "Model reduction via frequency weighted balanced realization," Control, Theory and Advanced Technology, vol. 8, no. 2, pp. 341-351, 1992.

[13] A. Varga and B. D. O. Anderson, "Accuracy-enhancing methods for balancing-related frequency-weighted model and controller reduction," Automatica, vol. 39, no. 5, pp. 919927, 2003.

[14] G. Wang, V. Sreeram, and W. Q. Liu, "A new frequencyweighted balanced truncation method and an error bound," IEEE Transactions on Automatic Control, vol. 44, no. 9, pp. 1734-1737, 1999.

[15] A. Ghafoor and V. Sreeram, "A survey/review of frequencyweighted balanced model reduction techniques," Journal of Dynamic Systems, Measurement and Control, Transactions of the ASME, vol. 130, no. 6, Article ID 061004, pp. 1-16, 2008.

[16] A. C. Antoulas, "An overview of model reduction methods and a new result," in Proceedings of the 48th IEEE Conference on Decision and Control Held Jointly with 28th Chinese Control Conference (CDC/CCC '09), pp. 5357-5361, December 2009.

[17] G. Genta and A. Gugliotta, "A conical element for finite element rotor dynamics," Journal of Sound and Vibration, vol. 120, no. 1, pp. 175-182, 1988.

[18] D. Combescure and A. Lazarus, "Refined finite element modelling for the vibration analysis of large rotating machines: application to the gas turbine modular helium reactor power conversion unit," Journal of Sound and Vibration, vol. 318, no. 4-5, pp. 1262-1280, 2008.

[19] A. Nandi, "Reduction of finite element equations for a rotor model on non-isotropic spring support in a rotating frame," Finite Elements in Analysis and Design, vol. 40, no. 9-10, pp. 935-952, 2004.
[20] E. Chatelet, F. D’Ambrosio, and G. Jacquet-Richardet, "Toward global modelling approaches for dynamic analyses of rotating assemblies of turbomachines," Journal of Sound and Vibration, vol. 282, no. 1-2, pp. 163-178, 2005.

[21] G. F. M. Lalanne, Rotordynamic Prediction in Engineering, John Wiley \& Sons, New York, NY, USA, 1998.

[22] T. Yamamoto and Y. Ishida, Linear and Nonlinear Rotordynamics, John Wiley \& Sons, New York, NY, USA, 2001.

[23] D. Childs, Turbomachinery Rotordynamics: Phenomena, Modeling and Analysis, John Wiley \& Sons, New York, NY, USA, 1993.

[24] J. Tonnesen and P. K. Hansen, "Some experiments on the steady state charactersitics of a cylindrical fluid-film bearing considering thermal effects," Journal of Lubrication Technology, Transaction of ASME, pp. 107-114, 1981.

[25] P. E. Allaire, "Rotor dynamics course notes," Tech. Rep., Rotating Machinery and Controls Lab, Univerity of Virginia, 2009.

[26] F. C. Nelson, "A brief history of early rotor dynamics," Journal of Sound and Vibration, vol. 37, no. 6, pp. 8-11, 2003.

[27] J. M. Vance, B. T. Murphy, and H. A. Tripp, "Critical speeds of rotating machinery: computer predictions vs. experimental measurements-part ii: effects of tilting pad bearings and foundation effects," ASME Journal of Vibration, Acoustics, Stress and Reliability in Design, vol. 109, pp. 8-14, 1987.

[28] H. D. Nelson and J. M. McVaugh, "The dynamics of rotor bearing systems using finite elements," Journal of Engineering for Industry, vol. 98, pp. 593-600, 1976.

[29] R. L. Ruhl and J. F. Booker, "Finite element model for distributed parameter turborotor systems," Journal of Engineering for Industry, Transactions of the ASME, vol. 94, no. 1, pp. 126-132, 1972.

[30] R. L. Eshelman and R. A. Eubanks, "On the critical speeds of a continuous rotor," ASME Journal of Engineering for Industry, vol. 91, pp. 1180-1188, 1969.

[31] H. D. Nelson, "A finite rotating shaft element using timoshenko beam theory," ASME Journal of Mechanical Design, vol. 102, no. 4, pp. 793-803, 1980.

[32] D. L. Thomas, J. M. Wilson, and R. R. Wilson, "Timoshenko beam finite elements," Journal of Sound and Vibration, vol. 31, no. 3, pp. 315-330, 1973.

[33] K. E. Rouch and J. S. Kao, "A tapered beam finite element for rotor dynamics analysis," Journal of Sound and Vibration, vol. 66, no. 1, pp. 119-140, 1979.

[34] T. A. Vest and M. S. Darlow, "Modified conical beam element based on finite element analysis. Experimental correlations," Journal of Vibration, Acoustics, Stress, and Reliability in Design, vol. 112, no. 3, pp. 350-354, 1990.

[35] A. Nandi, "On computation of response of a rotor in deformed configuration using three-dimensional finite elements," Communications in Numerical Methods in Engineering, vol. 19, no. 3, pp. 179-195, 2003.

[36] J. S. Rao and R. Sreenivas, "Dynamics of asymmetric rotors using solid models," in Proceedings of the International Gas Turbine Congress, 2003.

[37] A. Nandi and S. Neogy, "Modelling of rotors with threedimensional solid finite elements," Journal of Strain Analysis for Engineering Design, vol. 36, no. 4, pp. 359-371, 2001.

[38] R. W. Stephenson and K. W. Rouch, "Modeling rotating shafts using axisymmetric solid finite elements with matrix reduction," Journal of Vibration and Acoustics, Transactions of the ASME, vol. 115, no. 4, pp. 484-489, 1993. 
[39] E. Chatelet, D. Lornage, and G. Jacquet-Richardet, "A three dimensional modeling of the dynamic behavior of composite rotors," International Journal of Rotating Machinery, vol. 8, no. 3, pp. 185-192, 2002.

[40] M. Geradin and N. Kill, "A new approach to finite element modeling of flexible rotors," Engineering Computations, vol. 1, no. 1, pp. 52-64, 1984.

[41] D. M. Tran, "Component mode synthesis methods using interface modes. Application to structures with cyclic symmetry," Computers and Structures, vol. 79, no. 2, pp. 209-222, 2001.

[42] D. M. Tran, "Component mode synthesis methods using partial interface modes: application to tuned and mistuned structures with cyclic symmetry," Computers and Structures, vol. 87, no. 17-18, pp. 1141-1153, 2009.

[43] G. Jacquet-Richardet, G. Ferraris, and P. Rieutord, "Fequencies and modes of rotating flexible bladed disc-shaft assemblies: a global cyclic symmetry approach," Journal of Sound and Vibration, vol. 191, no. 5, pp. 901-915, 1996.

[44] I. Ansys, "Rotordynamic analysis guide," Tech. Rep., Swanson Analysis Systems, Inc., 2009.

[45] H. N. Ozguven and Z. Levent Ozkan, "Whirl speeds and unbalance response of multibearing rotors using finite elements," Journal of Vibration, Acoustics, Stress, and Reliability in Design, vol. 106, no. 1, pp. 72-79, 1984.

[46] L. M. Greenhill, W. B. Bickford, and H. D. Nelson, "A conical beam finite element for rotor dynamic analysis," Journal of Vibration, Acoustics, Stress, and Reliability in Design, vol. 107, no. 4, pp. 421-430, 1985.

[47] J. R. T. C. Gmur, "Shaft fnite elements for rotor dynamics analysis," ASME Journal of Vibration and Acoustics, vol. 113, pp. 482-493, 1993.

[48] R. W. Stephenson, K. E. Rouch, and R. Arora, "Modelling of rotors with axisymmetric solid harmonic elements," Journal of Sound and Vibration, vol. 131, no. 3, pp. 431-443, 1989.

[49] C. M. P. Bladh, "Component-mode-based reduced order modeling techniques for mistuned bladed disks_-part II: application," Journal of Engineering for Gas Turbines and Power, vol. 123, no. 1, pp. 100-108, 2001.

[50] C. M. P. Bladh, "Component-mode-based reduced order modeling techniques for mistuned bladed disks_-part 1 : theoretical models," Journal of Engineering for Gas Turbines and Power, vol. 123, no. 1, pp. 89-99, 2001.

[51] A. Nandi, S. Neogy, and A. S. Das, "Application of the harmonic balance technique to finite element models of asymmetric rotors via sparse kronecker products," Journal of Strain Analysis for Engineering Design, vol. 40, no. 6, pp. 549557, 2005.

[52] J. Sasek, M. Hajzman, and V. Zeman, "Modeling of rotating shafts with flexible disks," in Proceedings of 22nd Conference Computational Mechanics, 2006.

[53] D. S. Kumar, C. Sujatha, and N. Ganesan, "A modified semianalytical approach towards the modelling of a shaft-disc system," Computers and Structures, vol. 61, no. 1, pp. 189191, 1996.

[54] O. A. Bauchau, J. Rodriguez, and S. Y. Chen, "Coupled rotorfuselage analysis with finite motions using component mode synthesis," Journal of the American Helicopter Society, vol. 49, no. 2, pp. 201-211, 2004.

[55] R. W. Stephenson, Rotor system analysis: a coupled rotorfoundation matrix approach, Ph.D. thesis, 1990.

[56] Z. He, B. I. Epureanu, and C. Pierre, "Fluid-structural coupling effects on the dynamics of mistuned bladed disks," AIAA Journal, vol. 45, no. 3, pp. 552-561, 2007.
[57] M. A. Mohiuddin, M. Bettayeb, and Y. A. Khulief, "Dynamic analysis and reduced order modelling of flexible rotorbearing systems," Computers and Structures, vol. 69, no. 3, pp. 349-359, 1998.

[58] K. E. Rouch and J. S. Kao, "Dynamic reduction in rotor dynamics by the finite element method," Journal of Mechanical Design, Transactions of the ASME, vol. 102, no. 2, pp. 360$368,1980$.

[59] P. R. Houlston, S. D. Garvey, and A. A. Popov, "Modal control of vibration in rotating machines and other generally damped systems," Journal of Sound and Vibration, vol. 302, no. 1-2, pp. 104-116, 2007.

[60] Y. G. Jei and C. W. Lee, "Modal analysis of continuous asymmetrical rotor-bearing systems," Journal of Sound and Vibration, vol. 152, no. 2, pp. 245-262, 1992.

[61] Y. A. Khulief and M. A. Mohiuddin, "On the dynamic analysis of rotors using modal reduction," Finite Elements in Analysis and Design, vol. 26, no. 1, pp. 41-55, 1997.

[62] C. W. Lee, R. Katz, A. G. Ulsoy, and R. A. Scott, "Modal analysis of a distributed parameter rotating shaft," Journal of Sound and Vibration, vol. 122, no. 1, pp. 119-130, 1988.

[63] W. Wang and J. Kirkhope, "New eigensolutions and modal analysis for gyroscopic/rotor systems-part 1: undamped systems," Journal of Sound and Vibration, vol. 175, no. 2, pp. 159-170, 1994.

[64] A. Shanmugam and C. Padmanabhan, "A fixed-free interface component mode synthesis method for rotordynamic analysis," Journal of Sound and Vibration, vol. 297, no. 3-5, pp. 664-679, 2006.

[65] W. Wang and J. Kirkhope, "Component mode synthesis for damped rotor systems with hybrid interfaces," Journal of Sound and Vibration, vol. 177, no. 3, pp. 393-410, 1994.

[66] D. A. Glasgow and H. D. Nelson, "Stability analysis of rotorbearing systems using component mode synthesis," Journal of Mechanical Design, Transactions of the ASME, vol. 102, no. 2, pp. 352-359, 1980.

[67] E. M. ElBeheiry, "On eigenproblem solution of damped vibrations associated with gyroscopic moments," Journal of Sound and Vibration, vol. 320, no. 3, pp. 691-704, 2009.

[68] R. Ohayon, R. Sampaio, and C. Soize, "Dynamic substructuring of damped structures using singular value decomposition," Journal of Applied Mechanics, Transactions ASME, vol. 64, no. 2, pp. 292-298, 1997.

[69] W. Wang and J. Kirkhope, "Complex component mode synthesis for damped systems," Journal of Sound and Vibration, vol. 181, no. 5, pp. 781-800, 1995.

[70] M. R. Brake and J. A. Wickert, "Modal analysis of a continuous gyroscopic second-order system with nonlinear constraints," Journal of Sound and Vibration, vol. 329, no. 7, pp. 893-911, 2010.

[71] V. Barzdaitis, M. Bogdevicius, R. Didziokas, and M. Vasylius, "Modeling and diagnostics of gyroscopic rotor," Journal of Vibroengineering, vol. 11, no. 4, pp. 627-635, 2009.

[72] A. K. Samantaray, "Steady-state dynamics of a non-ideal rotor with internal damping and gyroscopic effects," Nonlinear Dynamics, vol. 56, no. 4, pp. 443-451, 2009.

[73] A. Y. Lee, "A krylov model reduction methodology for articulated, multi-flexible body structures," Tech. Rep., Jet Propulsion Laboratory at California Institute of Technology, 1994, http://trs-new.jpl.nasa.gov/dspace/bitstream/ 2014/32588/1/94-0334.pdf.

[74] U. Baur, C. A. Beattie, P. Benner, and S. Gugercin, "Interpolatory projection methods for parameterized model reduction," in Chemnitz Scientific Computing Preprints, 2009. 
[75] H. Panzer, J. Mohring, R. Eid, and B. Lohmann, "Parametric model order reduction by matrix interpolation," Automatisierungstechnik, vol. 58, no. 8, pp. 475-484, 2010.

[76] L. Daniel, O. C. Siong, L. S. Chay, K. H. Lee, and J. White, "A multiparameter moment-matching modelreduction approach for generating geometrically parameterized interconnect performance models," IEEE Transactions on Computer-Aided Design of Integrated Circuits and Systems, vol. 23, no. 5, pp. 678-693, 2004.

[77] V. Ganine, D. Laxalde, H. Michalska, and C. Pierre, "Parameterized reduced order modeling of misaligned stacked disks rotor assemblies," Journal of Sound and Vibration, vol. 330, no. 3, pp. 445-460, 2011.

[78] J. T. Sawicki and W. K. Gawronski, "Balanced model reduction and control of rotor-bearing systems," Journal of Engineering for Gas Turbines and Power, vol. 119, no. 2, pp. 456-463, 1997.

[79] G. W. Fan, H. D. Nelson, P. E. Crouch, and M. P. Mignolet, "LQR-based least-squares output feedback control of rotor vibrations using the complex mode and balanced realization methods," Journal of Engineering for Gas Turbines and Power, vol. 115, no. 2, pp. 314-323, 1993.

[80] Y. Chahlaoui, D. Lemonnier, A. Vandendorpe, and P. Van Dooren, "Second-order balanced truncation," Linear Algebra and Its Applications, vol. 415, no. 2-3, pp. 373-384, 2006.

[81] V. Sreeram and S. Sahlan, "Improved results on frequency weighted balanced truncation," in Proceedings of the 48th IEEE Conference on Decision and Control Held Jointly with 28th Chinese Control Conference (CDC/CCC '09), pp. 32503255, December 2009.

[82] C. A. Beattie and S. Gugercin, "A trust region method for optimal H model reduction," in Proceedings of the 48th IEEE Conference on Decision and Control Held Jointly with 28th Chinese Control Conference (CDC/CCC '09), pp. 5370-5375, December 2009.

[83] S. Gugercin, R. V. Polyuga, C. A. Beattie, and A. J. Van Der Schaft, "Interpolation-based $\mathrm{H}$ model reduction for port-Hamiltonian systems," in Proceedings of the 48th IEEE Conference on Decision and Control Held Jointly with 28th Chinese Control Conference (CDC/CCC '09), pp. 5362-5369, December 2009.

[84] R. Tasaltin, "Active vibration control strategies for a flexiblerotor bearing system," 1992.

[85] R. J. Guyan, "Reduction of stiffness and mass matrices," AIAA Journal, vol. 3, p. 380, 1965.

[86] R. D. Cook, D. S. Malkus, M. E. Plesha, and R. J. Witt, Concepts and Application of Finite Element Analysis, John Wiley \& Sons, New York, NY, USA, 4th edition, 2001.

[87] M. N. Sahinkaya, A. G. Abulrub, P. S. Keogh, and C. R. Burrows, "Multiple sliding and rolling contact dynamics for a flexible rotor/magnetic bearing system," IEEE-ASME Transactions on Mechatronics, vol. 12, no. 2, pp. 179-189, 2006.

[88] J. W. Lund, "Modal response of a flexible rotor in fluid film bearings," ASME Journal of Engineering for Industry, vol. 96, no. 2, pp. 525-533, 1974.

[89] A. A. Younan and A. El-Shafei, "Model calibration of anisotropic rotordynamic systems with speed-dependent parameters," Journal of Engineering for Gas Turbines and Power, vol. 130, no. 4, Article ID 042502, 2008.

[90] R. M. Laurenson, "Modal analysis of rotating flexible structures," AIAA Journal, vol. 14, no. 10, pp. 1444-1450, 1976.
[91] S. W. Hong and J. H. Park, "Modal analysis of multistepped distributed-parameter rotor-bearing systems using exact dynamic elements," Journal of Vibration and Acoustics, Transactions of the ASME, vol. 123, no. 3, pp. 401-403, 2001.

[92] P. Mahadevan, C. S. Jog, and A. Chatterjee, "Modal projections for synchronous rotor whirl," Proceedings of the Royal Society A, vol. 464, no. 2095, pp. 1739-1760, 2008.

[93] P. E. A. C. K. Choy and E. J. Gunter, "Damped critical speeds and unblance response by complex modal analysis," Tech. Rep., Rotating Machinery and Controls Lab, Univerity of Virginia, 1976.

[94] P. E. A. E. J. Gunter and C. K. Choy, "Modal analysis of turborotors using planar modes-theory," Tech. Rep., Rotating Machinery and Controls Lab, Univerity of Virginia, 1977.

[95] W. C. Hurty, "Dynamic analysis of structural systems using component modes," AIAA Journal, vol. 3, pp. 678-685, 1965.

[96] R. R. Craig and M. C. Bampton, "Coupling of substructures for dynamics analysis," AIAA Journal, vol. 6, pp. 1313-1319, 1968.

[97] P. Apiwattanalunggarn, S. W. Shaw, and C. Pierre, "Component mode synthesis using nonlinear normal modes," Nonlinear Dynamics, vol. 41, no. 1-3, pp. 17-46, 2005.

[98] B. Biondi and G. Muscolino, "Component-mode synthesis method variants in the dynamics of coupled structures," Meccanica, vol. 35, no. 1, pp. 17-38, 2000.

[99] D. F. Li and E. J. Gunter, "A study of the modal truncation error in the component analysis of a dual-rotor system," Tech. Rep., Rotating Machinery and Controls Lab, University of Virginia, 1980.

[100] R. Subbiah, R. Bhat, and T. Sankar, "Dynamic response of rotors using modal reduction techniques," Transactions of the ASME, Journal of Vibrations, Acoustics, and Reliability in Design, vol. 111, pp. 360-365, 1989.

[101] T. Iwatsubo, K. Shimbo, and S. Kawamura, "The study of nonlinear vibration analysis of rotor system using component mode synthesis method-(analysis using the harmonic balance method)," JSME International Journal Series C, vol. 45, no. 1, pp. 136-141, 2002.

[102] B. C. Moore, "Principal component analysis in linear systems-controllability observability and model reduction," IEEE Transactions on Automatic Control, vol. 26, no. 1, pp. 17-32, 1981.

[103] J. B. Burl, Linear Optimal Control: H2 and H Infinity Methods, Addison-Wesley, Reading, Mass, USA, 1998.

[104] M. Wamsler, "Retaining the influence of crucial local effects in mixed Guyan and modal reduction," Engineering with Computers, vol. 20, no. 4, pp. 363-371, 2005.

[105] J. L. Guyader, "Characterization and reduction of dynamic models of vibrating systems with high modal density," Journal of Sound and Vibration, vol. 328, no. 4-5, pp. 488506, 2009. 

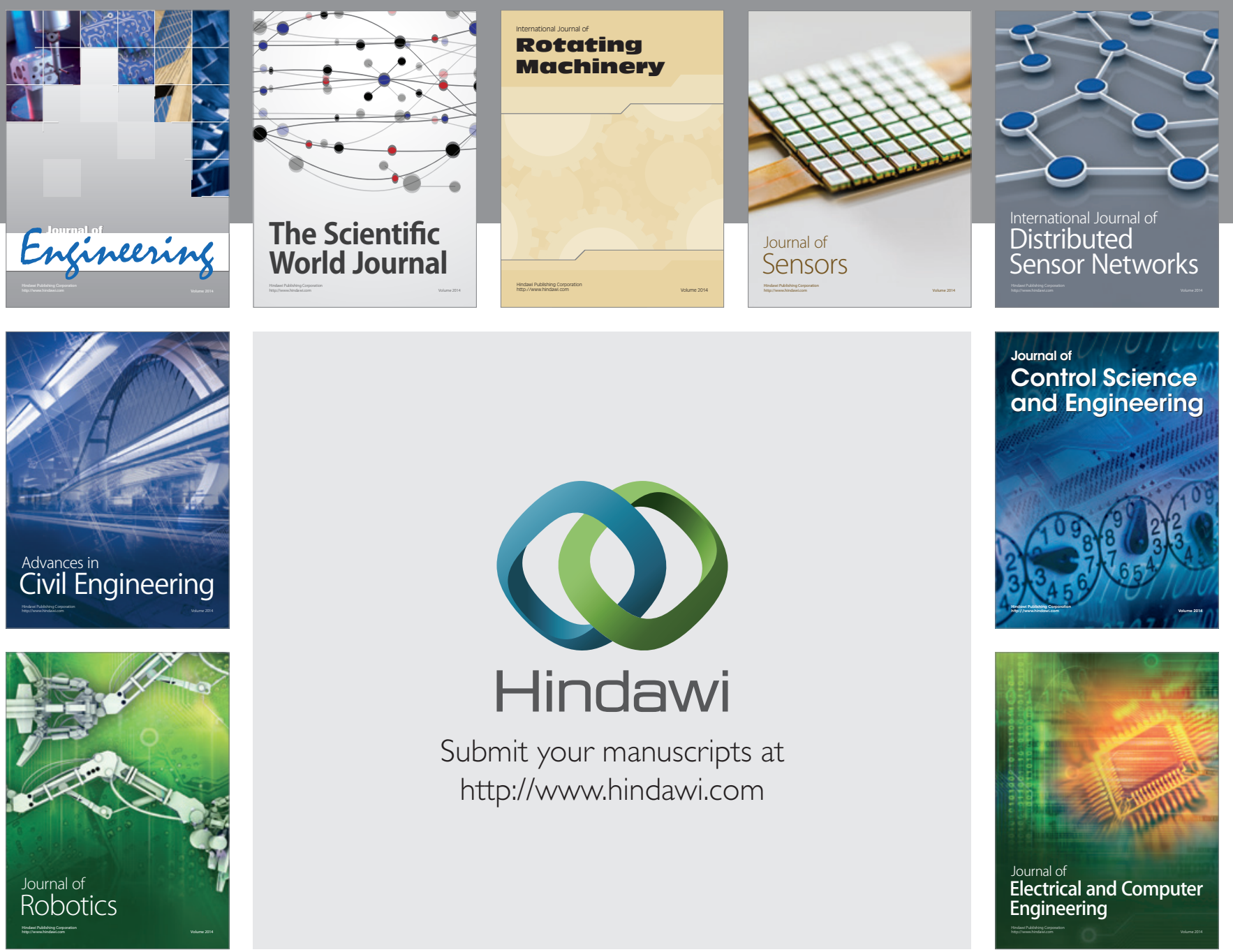

Submit your manuscripts at

http://www.hindawi.com
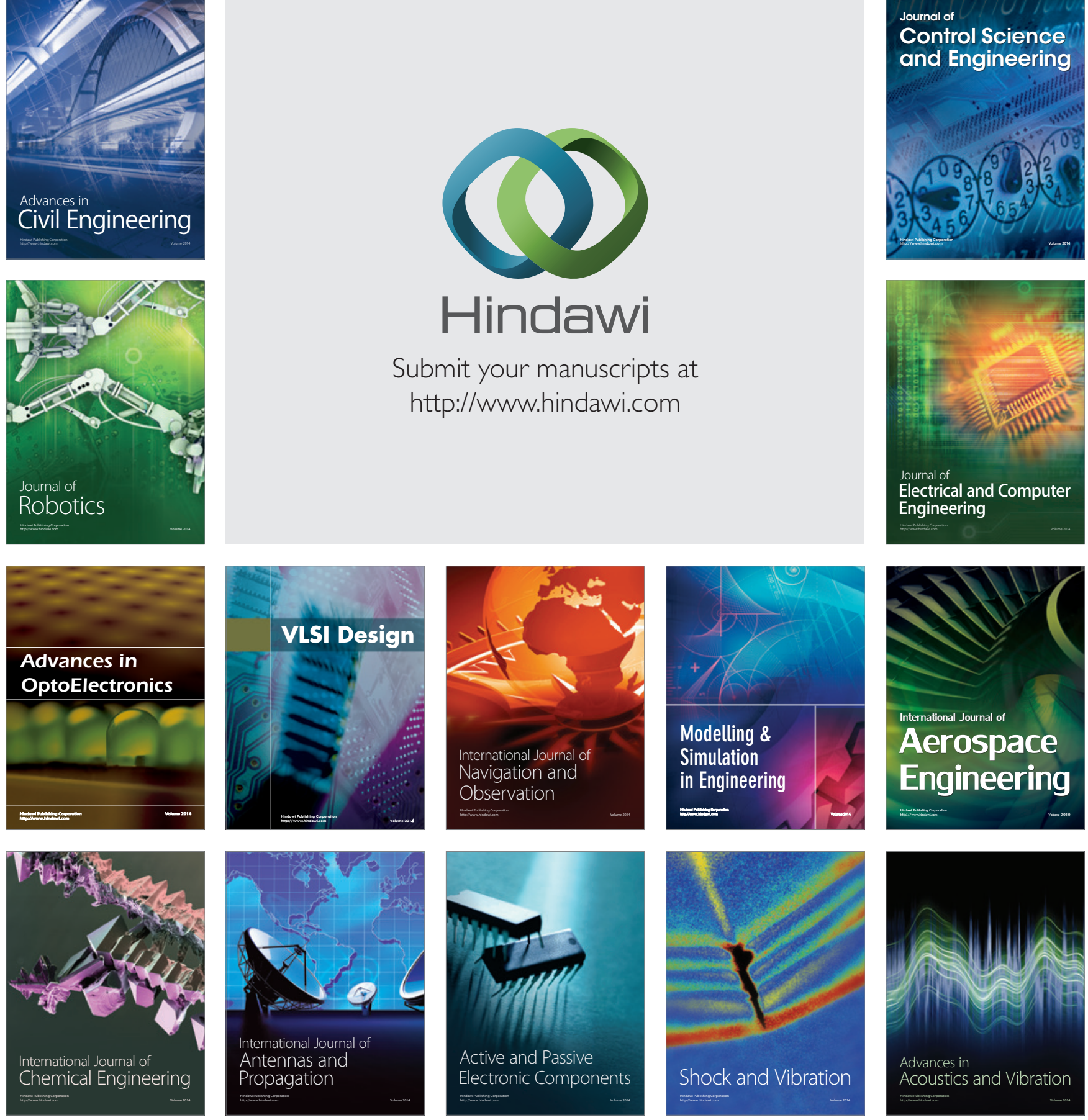